\section{Transoral robotic surgery (TORS) in South Africa}

To the Editor: A series of two-page advertisements have appeared in the September and November 2016 issues of the SAMJ, entitled 'da Vinci Transoral Robotic Surgery (TORS) is a minimally invasive alternative to open surgery and full-dose chemoradiation therapy for diseases of the head and neck. As these advertisements go beyond simply marketing surgical equipment, but seek to influence patients, referring doctors, oncologists and head and neck surgeons on how to treat cancers of the head and neck and sleep apnoea, we have taken the unusual step of writing to the editor of the SAMJ to correct some misconceptions in the advertisement.

The objectives of surgical resection of any cancer are to obtain clear surgical margins with acceptable morbidity. In the oropharynx, hypopharynx and larynx this can be achieved by transoral approaches, using either a headlight with electrocautery (as with a conventional tonsillectomy) or $\mathrm{CO}_{2}$ laser microsurgery, open surgical approaches, or TORS. TORS is simply a surgical tool that provides an excellent endoscopic view of the base of tongue, and has angled instruments to grab tissues and cut them with electrocautery. It is currently licensed by the US Food and Drug Administration (FDA) only to be used for T1 and T2 base-of-tongue cancers and may be particularly beneficial for selected, difficult-to-get-to cancers of the base of tongue. It has been widely adopted in the USA, but not in many other regions of the world owing to its very high cost. Purchasing a new TORS system is in the order of USD2 million, the annual maintenance costs are $\sim$ USD165 000, and the cost per case is USD15 000. Because of the high costs, hospitals have been known to encourage surgeons to use TORS to resect cancers, which can be more cheaply resected with $\mathrm{CO}_{2}$ laser or even with a headlight and cautery, to recover their investment.

However, most T1 and T2 base-of-tongue and oropharyngeal cancers can be resected with transoral $\mathrm{CO}_{2}$ laser, which is extremely cheap compared with TORS and is a well-established validated technique, also in South Africa (SA). Therefore, while TORS has benefits to resect base-of-tongue cancers in cases where $\mathrm{CO}_{2}$ laser does not provide adequate access, such cases are uncommon and the costs involved simply cannot be justified in the SA healthcare setting. Base-of-tongue cancers that cannot be resected by transoral $\mathrm{CO}_{2}$ laser can still be resected by suprahyoid or lateral pharyngotomy approaches, with minimal morbidity. Transoral $\mathrm{CO}_{2}$ laser resection has all the benefits of TORS listed in the advertisement, such as avoiding mandibulotomy, return to speech and swallowing, less blood loss, minimal scarring, and avoidance of tracheotomy.

The comment that TORS can reduce the requirement for chemoradiotherapy is to date not supported by results from randomised clinical trials. The majority of patients who undergo surgery for oropharyngeal cancers - especially that involving the base of tongue - will require adjuvant treatment to the postoperative tumour bed and/or the neck. If the tumour is excised with positive margins or the lymph nodes have extracapsular spread, many oncologists would still advocate chemoradiation. In theory, if a tumour of the base of tongue is excised, the postoperative radiotherapy target area may be smaller than if the tumour had not been excised. This in turn could result in at least some sparing of the pharyngeal constrictor muscles. However, this theoretical advantage would not be limited to TORS, but would apply to all the surgical techniques mentioned above.

Several randomised controlled trials are currently underway to better define the impact of TORS on the treatment of oropharyngeal cancers. In one such study the option of de-escalated treatment after TORS is being examined in patients with human papillomavirus (HPV)-positive tumours, while another study is comparing TORS with/without adjuvant treatment to chemoradiation in patients with HPV-negative tumours. Until the results of these and other studies are available, the role that TORS (or any surgery that achieves clear surgical margins) may or may not play in reducing the need for chemoradiotherapy is not known.

Therefore, even though TORS is an established surgical technique, it is extremely expensive in the SA context when there are cheaper alternatives available.

\section{Johannes J Fagan}

Professor and Chairman, Multidisciplinary Head and Neck Cancer Clinic, Groote Schuur Hospital and Division of Otolaryngology, Faculty of Health Sciences, University of Cape Town, South Africa

johannes.fagan@uct.ac.za

\section{Julie Wetter}

Radiation Oncologist, Multidisciplinary Head and Neck Cancer Clinic, Department of Radiation Oncology, Groote Schuur Hospital and Faculty of Health Sciences, University of Cape Town, South Africa

\section{Chris Joseph}

Honorary Associate Professor, Department of Otolaryngology, Faculty of Health Sciences, University of the Witwatersrand, Johannesburg; Senior Lecturer, Department of Otolaryngology, Faculty of Health Sciences, University of Pretoria; and Consultant Surgeon, Sandton Head and Neck Forum, Morningside Mediclinic, Johannesburg, South Africa

\section{Bernard Donde}

Radiotherapist, Sandton Head and Neck Forum, Morningside Mediclinic, Johannesburg, South Africa

S Afr Med J 2017;107(1):6. DOI:10.7196/SAMJ.2017.v107i1.12241 